CORRECTION

https://doi.org/10.1038/s41586-018-0721-y

\title{
Publisher Correction: Antibody and TLR7 agonist delay viral rebound in SHIV-infected monkeys
}

Erica N. Borducchi, Jinyan Liu, Joseph P. Nkolola, Anthony M. Cadena, Wen-Han Yu, Stephanie Fischinger, Thomas Broge, Peter Abbink, Noe B. Mercado, Abishek Chandrashekar, David Jetton, Lauren Peter, Katherine McMahan, Edward T. Moseley, Elena Bekerman, Joseph Hesselgesser, Wenjun Li, Mark G. Lewis, Galit Alter, Romas Geleziunas \& Dan H. Barouch

Correction to: Nature https://doi.org/10.1038/s41586-018-0600-6, published online 03 October 2018.

In Fig. $4 \mathrm{~b}$ of this Article, the $x$-axis labels 'PGT121' and 'GS-9620' were inadvertently swapped in both graphs. In addition, in Fig. $5 \mathrm{a}$ and b, 'TLR7' should have been 'GS-9620' for consistency. These figures have been corrected online. 